$$
\text { GA-A } \quad 20590
$$

JAN 131992

\title{
SOFTWARE UPGRADE FOR THE DIII-D NEUTRAL BEAM CONTROL SYSTEMS
}

\author{
by \\ J.W. CUMMINGS and P.A. THURGOOD
}

NOVEMBER 1991

\section{GENERAL ATOMICS}




\section{DISCLAIMER}

This report was prepared as an account of work sponsored by an agency of the United States Government. Neither the United States Government nor any agency thereof, nor any of their employees, makes any warranty, express or implied, or assumes any legal liability or responsibility for the accuracy, completeness, or usefulness of any information, apparatus, product, or process disclosed, or represents that its use would not infringe privately owned rights. Reference herein to any specific commercial product, process, or service by trade name, trademark, manufacturer, or otherwise, does not necessarily constitute or imply its endorsement, recommendation, or favoring by the United States Government or any agency thereof. The views and opinions of authors expressed herein do not necessurily state or reflect those of the United States Government or any agency thereof. 
GA-A20590

\title{
SOFTWARE UPGRADE FOR THE DIII-D NEUTRAL BEAM CONTROL SYSTEMS
}

\author{
by \\ J.W. CUMMINGS and P.A. THURGOOD
}

This is a preprint of a paper to be presented at the 14th Symposium on Fusion Engineering, September 30-October 3, 1991, San Diego, Califnrnia, and to be printed in the Proceedings.

\author{
Work supported by \\ Department of Energy \\ Contract DE-AC03-89ER51114
}
GENERAL ATOMICS PROJECT 3466
NOVEMBER 1991

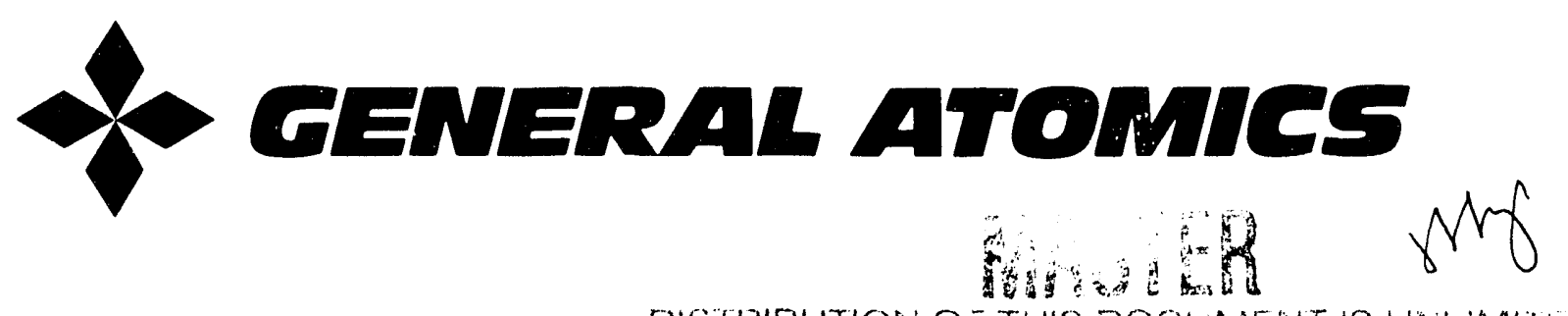




\title{
SOFTWARE UPGRADE FOR THE DIII-D NEUTRAL BEAM CONTROL SYSTEMS
}

\author{
J.W. Cummings and P.A. Thurgood \\ General Atomics \\ P.O. Box 85608 \\ San Diego, California 92186-9784
}

Abstract: The neutral beams are used to beat the plasma in the DIII-D tokamak, a fiusion energy research experiment operated by General Atomics (GA) and funded by the Department of Energy (DOE). The experiment is dedicated to demonstrating noninductive current drive of high beta high temperature divertor plasma with good confinement. The neutral beam heating system for the DIII-D tolamak uses four MODCOMP Classic computers for data acquisition and control of the four beamlines.

The Neutral Beam Software Upgrade project was launched in early 1990. The major goals were to upgrade the MAX IV operating system to the latest revision (K.1), use standard MODCOMP software (as much as possible), and to develop a very "user friendly," versatile system. Accomplishing these goals required new software to be developed and modifications to existing applications software to make it compatible with the latest operating system. The custom operating system modules to handle the message service and interrupt handling were replaced by the standard MODCOMP Inter Task Communication (ITC) and interrupt routines that are part of the MAX IV operating system. The message service provides the mechanism for doing shot task sequencing (task scheduling). The interrupt routines are used to connect external interrupts to the system. The new software developed consists of a task dispatcher, screen manager, and interrupt tasks. The existing applications software had to be modified to be compatible with the MODCOMP ITC services and consists of the Modcomp Infinity Data Base Manager, a multi-user system, and menu-driven operator system interface routines using the Infinity Data Base Manager.

\section{Introduction}

The neuiral beams are used to heat the plasma in the DIII-D tokamak. The DIII-D tokamak is a fusion energy research experiment operated by GA, funded by DOE.

This paper describes the Neutral Beam Software System upgrade project. This was a major project requiring system design, new software, and major modifications to existing software. This paper describes the overall neutral beam system hardware/software in general terms and the software system upgrade in detail in the following sections. The operational benefits and future plans for the systern will be discussed.

\section{System Functional Description}

The neutral beam computer systems are used to control the firing of the neutral beam shots and to acquire data from the shots. There are four beamlines each with two neutral beam sources. The eight sources are independent of each other. There are several modes of beamline operation and several ways of synchronizing the sources.

A sequence of programs (shot tasks) is run during each phase of a shot. A shot is also controlled by a set of "command language procedures" containing sequences of commands to control the shot. This allows the user to control the beamline hardware with a sequence of simple high level language commands. All of the shot tasks are synchronized by intertask messages routed through the dispatcher task. These shot tasks may do data acquisition, perform control functions, or both.

The shot task sequences to be sun are defined in Modcomp Infinity Data Base Manager files for each combination of mode and shot phase. Shot tasks may be preconditioned which ensures that the preconditioned task will not run until a certain task or combination of tasks has completed.

Data acquired during a shot is stored in the "run time" data base. This data is valid only for the current shot. This data may be displayed on the display monitors. Selected shot data is also stored in a Historical data base capable of storing 12,000 shots per source. A subset of the shot historical record is written to the DIII-D data acquisition computer at the end of each shot.

\section{System Confiquration}

MODCOMP Classic 7870 computer systems are used for data acquisition and control of the DIII-D neutral beamlines. Each beamline exaploys a MODCOMP Classic CPU connected to dual ported bard disks, semiconductor memory, interactive batch terminals, magnetic tape drive, CAMAC A/D hardware, Grinnell display terminals, CPU links, Macintosh (MAC) operator console, system console, and external interrupts. Figure 1 is a hardware diagram of the pertinent hardware for the NB 30 and NB 330 systems.

Software consists of the Modcomp MAX IV operating system, Modcomp Infinity Data Base Manager, Computer Network Interface software, and applications software. Software developed in-house includes the multi-user system, task dispatcher, screen manager, shot control and data acquisition tasks, external interrupt tasks, interactive operator software, command language sequencer, MAC operator console interface, historical data base software, and display software.

\section{New Software System Upgrade Goals}

The original goal was to upgrade MAX IV to the latest revision level changing whatever software necessary to implement the upgrade. After extensive investigation, it became apparent that major changes would be required to accomplish this. The upgrade plan allowed for considerable system operational enhancements as well as upgrading the operating system. The upgrade project got underway in early 1990. The main goals of the upgrade plan were to install latest revision of the MAX IV operating system, develop new software that is both "user-friendly" and flexible, use standard Modcomp software packag's as much as possible, and maintain compatibility with the other Modcomp systems. 


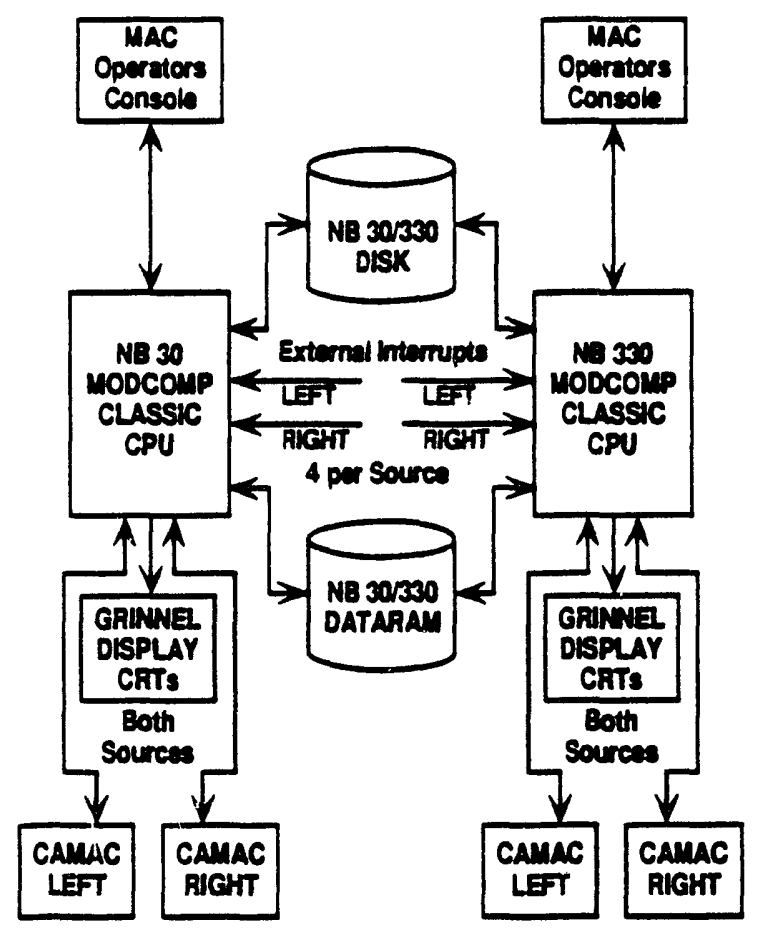

Fig. 1. NB 30/330 systems hardware diagram.

\section{New Software System Description}

The new software can be divided into two parts. The first part is the new software that upgraden the system and enhances it. The second part is software that had to be changed in order to be compatible with the first part. This section will deal only with the former. The MAX IV upgrade and ITC will not be discussed as they are part of the standard MODCOMP system.

A multi-user system was developed in-house and has been in use on a development system since late 1987. The multi-user software consists of a "sign on" task and supporting software to make the MODCOMP appear more like a time-sharing system to the interactive users. This enhanced development system was a great aid in completing the large number of software tasks.

The Infinity Data Base Manager is a standard MODCOMP software product supplied by a 3rd party vendor. At the present time, it replaces the old productions data base used by the dispatcher and screen manager. Adding Infinity yields a robust data base manager that is a standard product. Eventually, all data bases will be replaced with Infinity.

The new dispatcher and screen manager were the major part of the software upgrade. They were developed in-house and are completely different from the old software. The screen manager can now be thought of as an extension of the dispatcher. It is a separate task to enable the use of the queueing facility provided by ITC. The dispat: ther is used to route all messages between tasks. The dispatcher functions like a task scheduler using incoming and outgoing messages combined with a set of internal tables to determine what and how each task shall run. The internal tables are loaded from a set of Infinity Data Base files loaded during system startup. They may be reloader at any time by reinitializing the dispatcher except during a shot. The dispatcher responds to what the user has defined in the incoming message and data base files. All dispatcher messages contain an event type that determines what action should be taken. The action can be simply routing the message to the target task (Start Event) to running task sequences (Interrupt Event). The dispatcher is essentially generic allowing it to be used on any system with the MODCOMP Infinity Data Base Manager.

A powerful set of interactive software was developed to interface with the Infinity Data Base files. The software is menu driven making it "user friendly" as well as flexible. There are general interactive routines as well as special ones for manipulating selected sets of data bases. At present, there are two special purpose programs to interface to the dispatcher files and ITC port and server files.

The new external interrupt handling software consists of separate tasks connected to each of the external interrupts (eight per CPU). The task is activated by the interrupt which, in turn, sends a message to the dispatcher task. The content of the message sent to the dispatcher is the only difference between the interrupt tasks. The message sent is an "Interrupt Event" with the type of interrupt contained in the message. The "GET READY" interrupt task sends an "Initial Event" which is a special type of interrupt event telling the dispatcher to initialize all of its dynamic tables.

The new system software provides a flexible "user friendly" system. Any changes to the dispatcher data base files may be made at any time and loaded into the system in a matter of seconds. This allows the user to make changes during operations between shots. Figure 2 illustrates the function of the dispatcher and the data flow between it and other tasks.

\section{Neutral Beams Operational Benefits}

The software upgrade has provided many benefits to the operation of DIII-D neutral beam sources. The primary gains are "user friendliness," flexibility, and speed. Tests indicate at least a $15 \%$ reduction in total elapsed time for a shot, and thus data is available to the operator sooner.

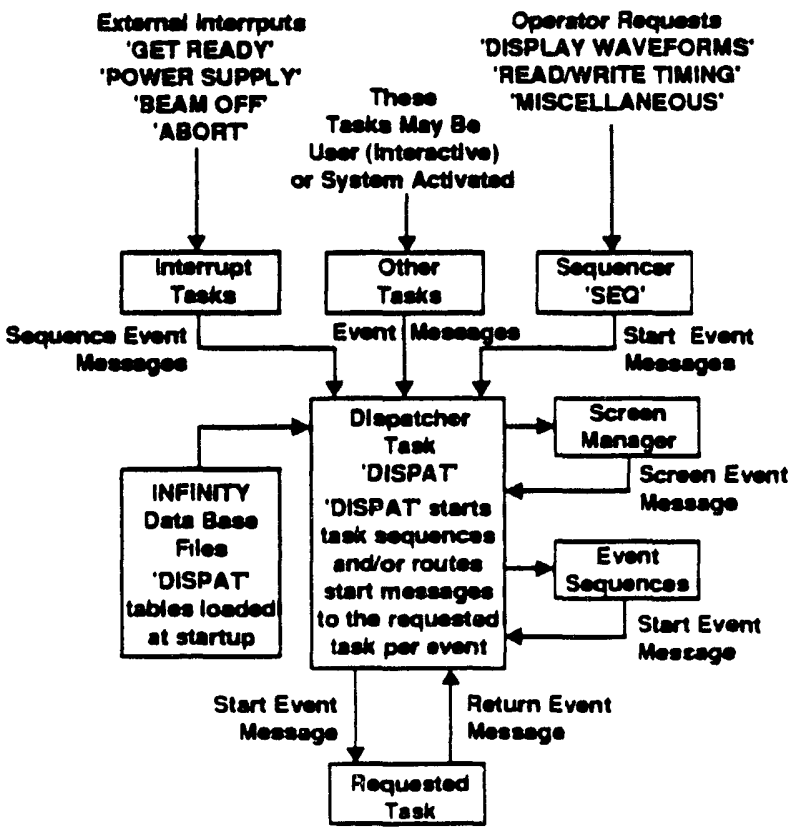

Fig. 2. Dispatcher function and data flow. 
Operational efficiency has been vastly improved by the new systems "user friendliness" and flexibility. "User friendliness" is the ease of interfacing to the system provided by the interactive menu driven software. A user may define or modify shot operational parameters easily without needing specialized knowledge of the computer system. The new system provides the flexibiiity to make these changes at any time it is desired. In the past, modifications to shot task options or sequences could not be made while running shots. They were difficult to make and required that the system be off-line for at least $45 \mathrm{~min}$. With the present system, modifications may be made in a matter of seconds and put into effect immediately. This allows modifications to be made during shots with the system on-line. Beam operation decisions may be made and implemented at any time. There are more shot task options available allowing more precise shot operation. Shot tasks may be easily run individually using a special interactive program. This provides more flexibility for testing. One of the most important benefits gained is that the system may be easily enhanced allowing for future operational benefits.

\section{Future Plans}

Now that the new system is in place, its capability may be greatly enhanced. Future plans are to replace more of the remaining software, namely the "Run Time" data base with new software that uses the Infinity Data Base manager. That will provide a platform for future enhancements. For example, the waveform display timing could be automatically adjusted to reflect the digitizer settings. A new task will be added to the MODCOMP that allows direct communication (bidirectional) to the operators console MAC.

\section{Acknowledgements}

This work was sponsored by the U.S. Department of Energy under Contract No. DE-AC03-89ER51114. 

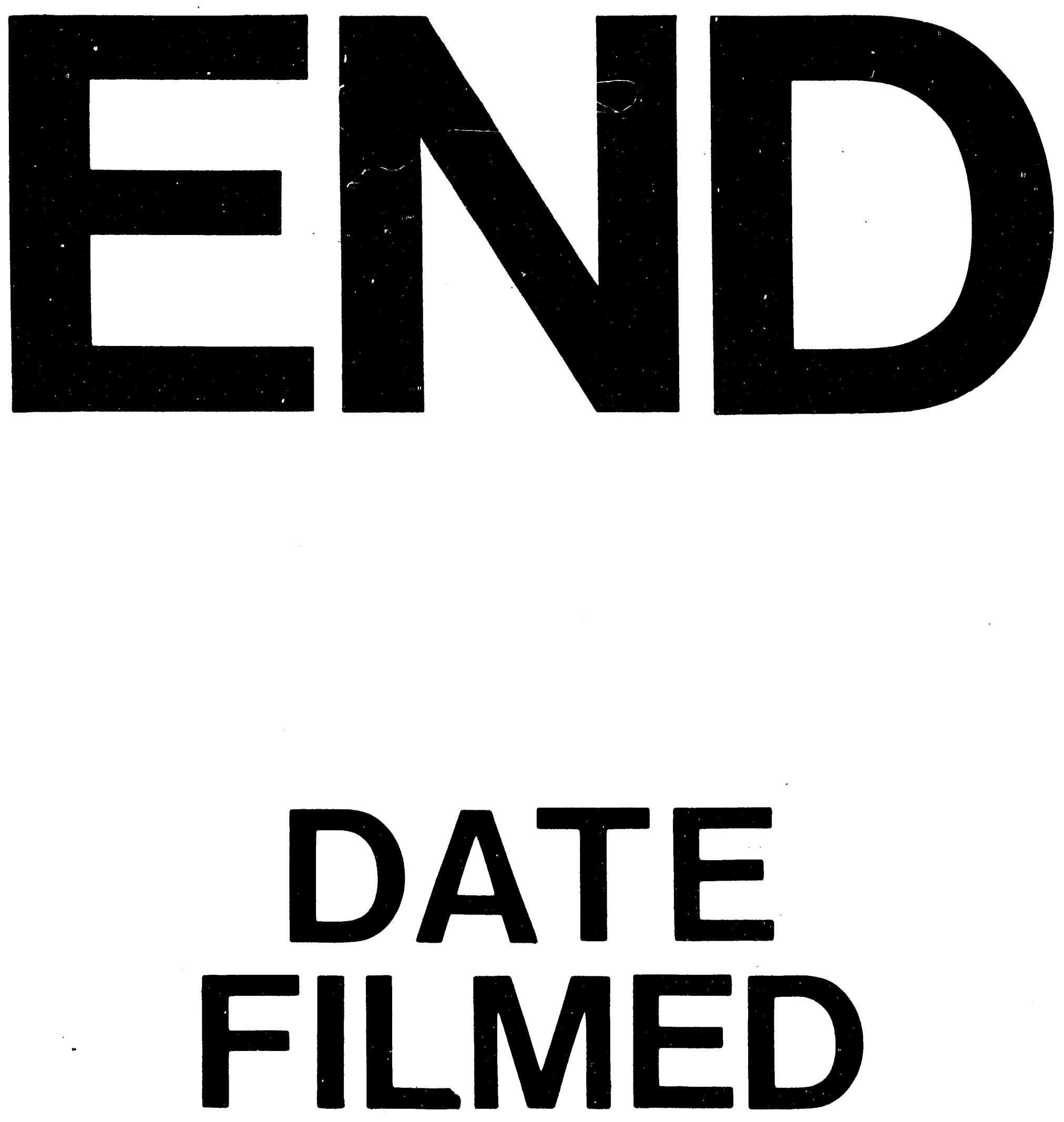

1

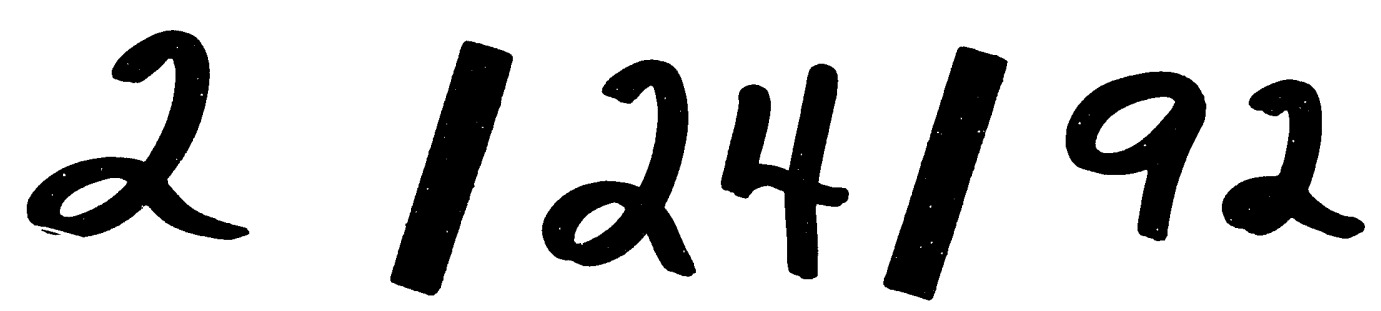




$$
\text { - }
$$

